\title{
The Melting Temperature of a Thermally Reversible Gel. III. Poly(vinyl alcohol)-Water Gels
}

\author{
Akira TAKAHASHI* and Sei-sho HiRAmitsu** \\ Department of Industrial Chemistry, Mie University, \\ Kamihama-cho, Tsu, Mie, 514 Japan.
}

(Received July 30, 1973)

\begin{abstract}
The gel melting temperatures of thermally reversible poly(vinyl alcohol) [PVA]-water gels were determined using the six conventional PVA samples, which differ in molecular weight. As previously observed in other thermally reversible gels, a linear relationship between the reciprocal absolute melting temperature $\left(T_{\mathrm{m}} \mathrm{g}\right)$ and the logarithm of $v_{2} x$ was found for each PVA gel, where $v_{2}$ is the volume fraction of polymer and $x$ the degree of polymerization. The results were analysed using the theoretical expression given in a previous paper. The analysis leads to the conclusion that the "cross-links" in the PVA gels consist of 7 syndiotactic units irrespective of $x$. It was also shown that the conclusion is in accordance with the sequence length estimated by Shibatani from the rubber-like elastic properties of PVA-water gels.

KEY WORDS Gel / Melting Temperature / Poly(vinyl alcohol) /

Thermally Reversible Gel / Sequence Length /
\end{abstract}

In the first paper of this series, ${ }^{1}$ the following equation was derived for the melting temperature $\left(T_{\mathrm{m}}^{\mathrm{g}}\right)$ of a thermally reversible gel, in which the "cross-links" in the gel are "crystallites"

$$
\begin{aligned}
\frac{1}{T_{\mathrm{m}}^{\mathrm{g}}}= & \frac{\zeta}{\Delta h_{\mathrm{u}} \zeta+B^{\prime} V_{\mathrm{A}} \zeta-2 \sigma_{\mathrm{ec}}}\left(\frac{\Delta h_{\mathrm{u}}}{T_{\mathrm{m}}}+\frac{R V_{\mathrm{A}}}{V_{1}}-R \ln X_{\mathrm{A}}\right) \\
& -\frac{R}{\Delta h_{\mathrm{u}} \zeta+B^{\prime} V_{\mathrm{A}} \zeta-2 \sigma_{\mathrm{ec}}} \ln v_{2} x
\end{aligned}
$$

In this equation, $v_{2}$ is the volume fraction of polymer in the gel, $x$ is the degree of polymerization of the polymer, ${ }^{*} \zeta$ is the number of crystallized units $A$ which enter into the crystallite as a sequence, $\Delta h_{\mathrm{u}}$ is the heat of fusion per repeating unit, $T_{\mathrm{m}}{ }^{0}$ is the equilibrium melting temperature of the polymer, $X_{\mathrm{A}}$ is the mole fraction of the crystallizable unit in the polymer, $B^{\prime}$ is the interaction energy density for the polymer-diluent pair, $\sigma_{\mathrm{ec}}$ is the interfacial free energy per crystalline sequence, and $V_{\mathrm{A}}$ and $V_{1}$ are the molar volumes of the unit

* To whom correspondence should be addressed.

** Department of Synthetic Chemistry, Nagoya University, Nagoya, Japan.

* For polydisperse polymer systems, $x$ in eq 1 must be replaced by the weight average degree of polymerization.
A and the solvent.

Equation 1 has the same form as that reported by Eldridge and Ferry. $^{2}$ However, eq 1 indicates the physical meanings of the intercept and slope in the plot of $1 / T_{\mathrm{m}}{ }^{\mathrm{g}}$ against $\ln v_{2} x$ more clearly than the Eldridge and Ferry equation does. Thus, we have examined eq 1 using both poly(vinyl chloride)-organic solvent gels $^{1}$ and ethylene vinyl acetate copolymer-organic solvent gels. $^{3}$ We found that eq 1 is quite satisfactory for representing the $T_{\mathrm{m}} \mathrm{g}$ of these gels as functions of $v_{2}, x, X_{A}$, and $B^{\prime}$. The crystallite size could also be estimated from the analysis of the experimental data according to eq 1 , and the $\zeta$ thus estimated was favorably compared with the crystalline sequence length determined by NMR spectroscopy for the polymers.

It is well known that the poly(vinyl alcohol) [PVA]-water system exhibits thermally reversible gelation, and the phenomenon has been studied by many workers. In their work, however, the measurements of the melting temperature of PVA gels were only carried out at constant molecular weight. Moreover, the stereoregularity of PVA, i.e., its copolymeric character was not taken into consideration. Thus, in 


\section{A. Takahashi and S. Hiramitsu}

their analysis of the melting temperature of the PVA gels, they relied upon the Eldridge and Ferry equation at constant molecular weight, particularly, to estimate the energy $\left(\Delta H_{\mathrm{m}}\right)$ associated with the dissociation of a "cross-link" in the gel. Maeda, Kashiwagi, and Kawai, ${ }^{4}$ as well as Hirai ${ }^{5}$ and also Haas, ${ }^{6}$ obtained $\Delta H_{\mathrm{m}}=$ $8.8 \mathrm{kcal} / \mathrm{mol}$ of "cross-link." A direct comparison between the value of $\Delta H_{\mathrm{m}}$ and the hydrogen bonding energy $=5-6 \mathrm{kcal} / \mathrm{mol}$ leads to the conclusion that a "cross-link" corresponds to one or two intermolecular hydrogen bonds formed from hydroxyl groups; however, from the more detailed studies of dilatancy of PVA solutions, Maeda, Kashiwagi, and $\mathrm{Kawai}^{7}$ have arrived at the conclusion that a "cross-link" has a "micellar-like structure," which consists of a fairly large number of succesive hydrated hydroxyl groups of PVA chains. Unfortunately, however, they could not estimate the size or length of the micell.

Very recently, Shibatani ${ }^{8}$ has determined the number of "cross-links" in a unit volume of PVA gel from the measurement of its rubberlike elastic property, and concluded that the "cross-link" consists of $6-8$ syndiotactic units in the PVA chains. Moreover, he has thrown a doubt upon the applicability of the Eldridge and Ferry equation to the PVA-water systems. Thus, there are still different opinions about the nature of the "cross-link." As we have pointed out previously, the Eldridge and Ferry equation must be regarded as an empirical equation; therefore, the conclusion that has been reached from the apparent $\Delta H_{\mathrm{m}}$ must be reexamined.

The purpose of this paper is to use the new data of $T_{\mathrm{m}}^{\mathrm{g}}$ of PVA gels which will be reported here to estimate the crystalline sequence length in the "cross-link" according to eq 1, and thus to clarify the nature of the "cross-link."

\section{EXPERIMENTAL}

\section{Materials}

Six samples of PVA which differ in molecular weight have been used. All the polymers were purified by dissolving them in hot water and precipitating the solutions in an acetonemethanol mixture, and were dried in vacuo for three days. The analysis showed that the mol\% of residual acetate group in each PVA sample was less than 0.01. Deionized water was used as solvent for both purification and gelation.

\section{Characterization of Polymers}

Molecular weights of the PVA samples were determined by viscosity measurements in water at $30^{\circ} \mathrm{C}$ using the viscosity equation ${ }^{9}$

$$
[\eta]=6.66 \times 10^{-4} M^{0.64}
$$

To determine the syndiotacticity of the samples, the ratio of optical density at $916 \mathrm{~cm}^{-1}$ to that at $849 \mathrm{~cm}^{-1}$ was estimated from the infrared spectrum of PVA film. The syndiotactic diads were calculated from the ratio by using the equation given by Murahashi, Nozakura, Sumi, and Hatada. ${ }^{10}$ The 1,2-glycol content was determined by the consumption of periodic acid according to the method reported by Ito, Noma, and Sakurada. ${ }^{11}$ Details of the six samples are given in Table I.

Table I. Degree of polymerization, syndiotacticity, and 1,2-glycol content in PVA

\begin{tabular}{cccc}
\hline $\begin{array}{c}\text { Sample } \\
\text { No. }\end{array}$ & DP & $\begin{array}{c}\text { Synd, a } \\
\%\end{array}$ & $\begin{array}{c}1,2-G l y c o l \\
\text { content, mol\% }\end{array}$ \\
\hline 1 & 632 & 54.6 & 2.21 \\
2 & 1250 & 56.8 & 2.23 \\
3 & 1364 & 60.9 & 2.25 \\
4 & 1682 & 57.1 & 2.22 \\
5 & 1955 & 56.7 & 2.25 \\
6 & 2250 & 53.0 & 2.24 \\
\hline
\end{tabular}

a Syndiotacticity in the diad.

\section{Melting Temperature and X-Ray Diffraction of Gel}

The methods used to determine the melting temperatures of thermally reversible gels were fully described in the previous papers. ${ }^{1,3}$ An aqueous solution of PVA in a gel melting temperature apparatus, which has also been described previously, ${ }^{1}$ was heated at $90^{\circ} \mathrm{C}$ for $1 \mathrm{hr}$, and then gelation of the PVA solution was conducted at $0^{\circ} \mathrm{C}$ for $24 \mathrm{hr}$. The X-ray diffraction patterns of the PVA gels, the dried PVA film prepared from the gel and water were obtained by the same procedure employed in the previous papers. ${ }^{1,3}$ 


\section{RESULTS AND DISCUSSION}

In Figure 1, the reciprocals of the gel melting temperatures are plotted against $\ln v_{2} x$ for three PVA gels. Since the plots are linear, it is apparent that eq 1 does hold in this case, too. For the other PVA gels, good straight lines were also obtained. The intercepts and the slopes determined from these plots are summarized in Table II.

The results were analysed as follows. By making use of the definitions given in the dilute solution treatment ${ }^{12}$

$$
\chi_{1}=\frac{B^{\prime} V_{1}}{R T}
$$

and

$$
\frac{1}{2}-\chi_{1}=\psi_{1}-\kappa_{1}=\psi_{1}\left(1-\frac{\Theta}{T}\right)
$$

eq 1 may be rewritten in the form

$$
\begin{aligned}
\frac{1}{T_{\mathrm{m}}{ }^{\mathrm{g}}}= & \frac{\zeta}{\Delta h_{\mathrm{u}} \zeta+R\left(V_{\mathrm{A}} / V_{1}\right) \phi_{1} \Theta \zeta-2 \sigma_{\mathrm{ec}}} \\
& \times\left[\frac{\Delta h_{\mathrm{u}}}{T_{\mathrm{m}}{ }^{0}}+\frac{R V_{\mathrm{A}}}{V_{1}}\left(\frac{1}{2}+\psi_{1}\right)-R \ln X_{\mathrm{A}}\right] \\
& -\frac{R}{\Delta h_{\mathrm{u}} \zeta+R\left(V_{\mathrm{A}} / V_{1}\right) \phi_{1} \Theta \zeta-2 \sigma_{\mathrm{ec}}} \ln v_{2} x
\end{aligned}
$$

In these equations, $\chi_{1}$ is the interaction parameter, $\phi_{1}$ the entropy of dilution parameter, $\kappa_{1}$ the corresponding enthalpy parameter, and $\Theta$ the Theta temperature. Dieu ${ }^{13}$ has reported that, for PVA in water at $298^{\circ} \mathrm{K}, \phi_{1}=-0.142, \kappa_{1}=$ -0.173 , and for the PVA-water system, $\Theta=$ $370^{\circ} \mathrm{K}$. Strictly speaking, the Flory-Huggins theory cannot be applied to the case where both $\psi_{1}$ and $\kappa_{1}$ are negative. However, we have assumed that the form of the theory is still applicable even in such a case. Hence, $\psi_{1}=$ -0.142 and $\Theta=370^{\circ} \mathrm{K}$ were employed in the analysis. We must also know both $\Delta h_{\mathrm{u}}$ and $T_{\mathrm{m}}{ }^{0}$. Sakurada, Nakajima, and Takita $^{14}$ reported that $\Delta h_{\mathrm{u}}=2630 \mathrm{cal} / \mathrm{mol}$ for a conventional PVA.

Since PVA crystallite has a hydrogen-bonded structure, it may be argued that the heat of fusion $=2630 \mathrm{cal} / \mathrm{mol}$ is too small when compared with the rupture energy $6 \mathrm{kcal} / \mathrm{mol}$ of $\mathrm{O}-\mathrm{H}$... O hydrogen bond. The heat of fusion $\left(\Delta H_{\mathrm{f}}\right)$ of crystals of alcohols, which are inter-

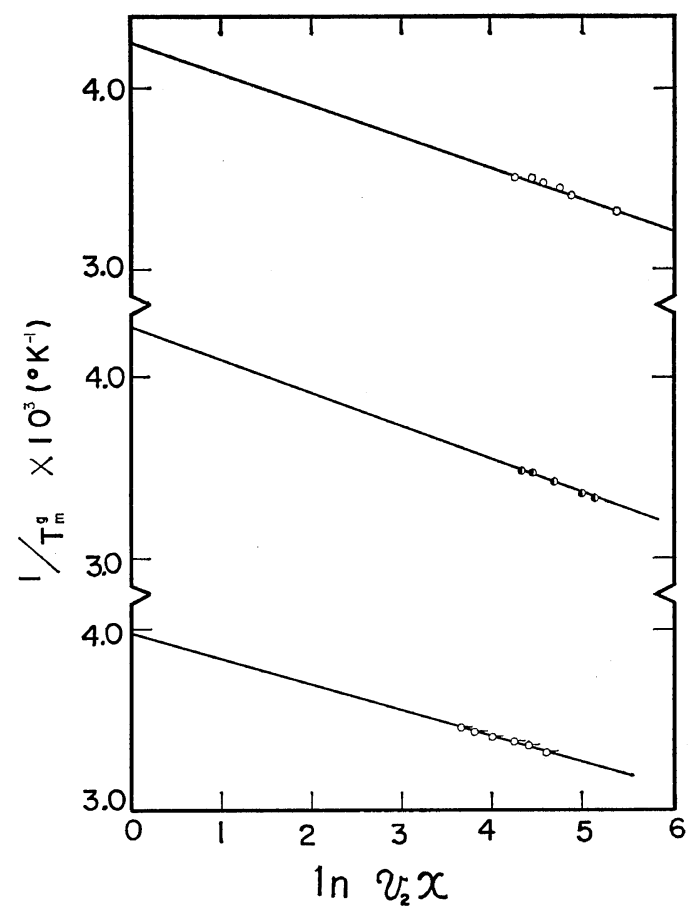

Figure 1. Plots of $1 / T_{\mathrm{m}} \mathrm{g} v s . \ln v_{2} x$ for three PVA gels. Degree of polymerization $(x): \bigcirc-, 632 ; 0$, 1682; $\bigcirc, 2250$.

molecularly hydrogen-bonded, are only slightly abnormal: i.e., for methanol, $\Delta H_{\mathrm{f}}=757 \mathrm{cal} / \mathrm{mol}$ and for ethanol, $\Delta H_{\mathrm{f}}=1200 \mathrm{cal} / \mathrm{mol}$. Pauling ${ }^{15}$ has already discussed the reasons for this and concluded that loss of energy due to the rupture of hydrogen bonds does not occur on fusion, since the liquid also contains the hydrogenbonded long chain or ring structures. For the same reason, $\Delta H_{\mathrm{u}}=2630 \mathrm{cal} / \mathrm{mol}$ for PVA is not abnormally low.

Sumi, Matsumura, Ohno, Nozakura, and Murahashi ${ }^{16}$ measured the melting temperatures of syndiotactic PVA. Of course, these melting temperatures are not the equilibrium melting temperatures, but, it was quite difficult to extrapolate the melting temperature data to that of 100-\% syndiotactic PVA, i.e., to obtain $T_{\mathrm{m}}{ }^{0}$. Therefore, we adopted $T_{\mathrm{m}}=599^{\circ} \mathrm{K}$, reported by them for the highest syndiotactic PVA, as $T_{\mathrm{m}}{ }^{0}$. Since it has been reported that predominantly isotactic PVA easily dissolves in cold water, ${ }^{17}$ we have only considered the melting temperature of syndiotactic PVA. The syndiotactic diads of 


\section{A. Takahashi and S. Hiramitsu}

the samples were treated as $X_{A}$. Making use of these values in eq 5, and following the authors' previously reported procedure, ${ }^{1}$ one may determine $\zeta$ and $\sigma_{\text {ec }}$ from the observed intercept and slope, respectively. The results are summarized in Table II.

The values of both $\zeta$ and $\sigma_{\mathrm{ec}}$ are almost constant for the six PVA samples. The average value of $\zeta$ is about seven. Again considering the fact that isotactic PVA dissolves in cold water, we may conclude that the "cross-links", in the PVA gels consist of sequences of seven syndiotactic units. From measurements of the rubber-like elastic properties of PVA gels, Shibatani ${ }^{8}$ has estimated that the syndiotactic sequence lengths in the "cross-link" are about 6-8. He has arrived at the conclusion from the measured cross-linking density in PVA gels, whereas we have arrived at the same conclusion

Table II. The estimation of $\zeta$ and $\sigma_{\mathrm{ec}}$

\begin{tabular}{ccccc}
\hline \multicolumn{5}{c}{ Obsd } \\
\cline { 2 - 4 } Sample & $\begin{array}{c}\text { Intercept } \\
\times 10^{3},{ }^{\circ} \mathrm{K}^{-1}\end{array}$ & $\begin{array}{c}\text { Slope } \\
\times 10^{4},{ }^{\circ} \mathrm{K}^{-1}\end{array}$ & $\zeta$ & $\sigma_{\mathrm{ec}}, \mathrm{cal} / \mathrm{mol}$ \\
\hline 1 & 3.98 & 1.30 & 8.4 & 2610 \\
2 & 4.18 & 1.77 & 6.5 & 2320 \\
3 & 4.38 & 2.24 & 5.5 & 2700 \\
4 & 4.31 & 1.90 & 6.3 & 2420 \\
5 & 4.24 & 1.76 & 6.7 & 2480 \\
6 & 4.20 & 1.60 & 7.1 & 2460 \\
\hline \multicolumn{5}{c}{ Average } \\
\hline
\end{tabular}

from the analysis of the melting temperature of PVA gels, which is concerned with the free energy of formation of the "crystallite" in the gel. Thus, the two independent studies are different in their theoretical approaches. Since both methods gave excellent agreement in the estimation of the sequence lengths, we may conclude that the present analysis of the gel melting temperature is valid.

The syndiotactic diads and 1,2-glycol content in the PVA samples are almost the same; moreover, almost the same values of $\zeta$ and $\sigma_{\text {ec }}$ have been obtained; then, neglecting the minor variations in the values of $X_{\mathrm{A}}, \zeta$, and $\sigma_{\mathrm{ec}}$, we may

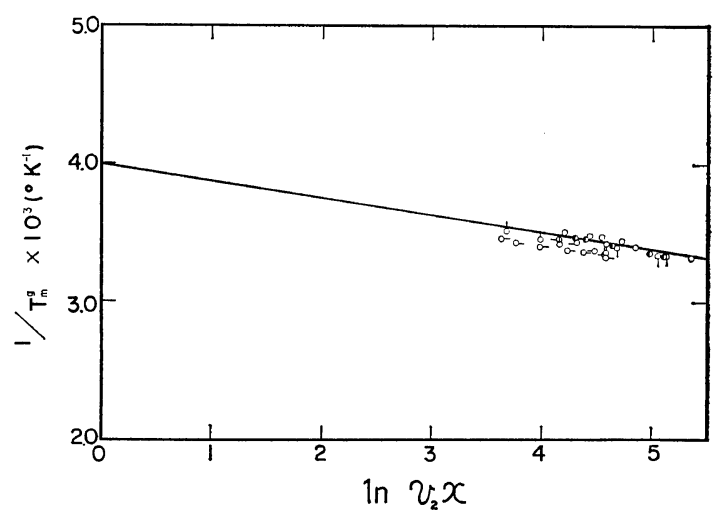

Figure 2. $1 / T_{\mathrm{m}}^{\mathrm{g}}$ plotted against $\ln v_{2} x$ for all the PVA gels. Besides the symbols used in Figure 1, the degrees of polymerization are: $\bigcirc, 1250 ;-\bigcirc$, 1364;, 1955.

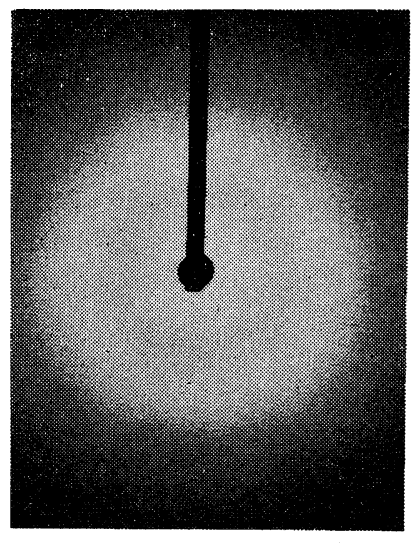

(a)

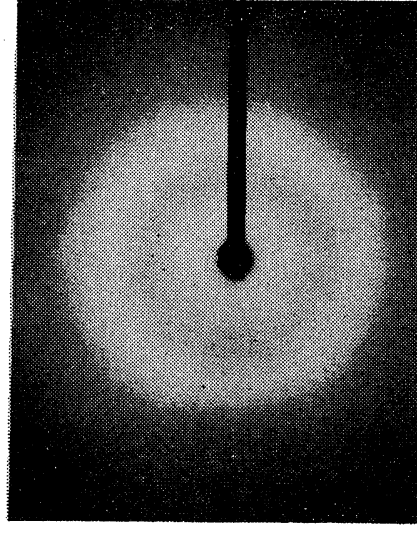

(b)

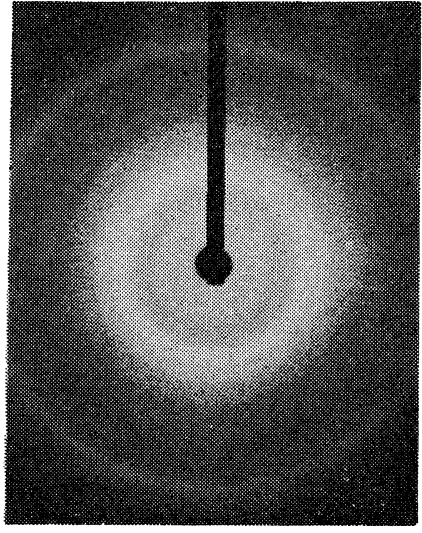

(c)

Figure 3. X-ray diffraction patterns of: (a) PVA gel (sample no. $4, C=25 \mathrm{~g} / \mathrm{l}$ at $15^{\circ} \mathrm{C}$ ), (b) water, (c) PVA film prepared from the gel. 
expect that the $1 / T_{\mathrm{m}}{ }^{\mathrm{g}} v s . \ln v_{2} x$ plots for all the PVA gels examined herein will be reduced almost on a straight line. In Figure 2, the plots are shown. It is clear that this expectation is almost fulfilled. Incidentally, it was observed that Maeda, et al.'s data also fall on the straight line in the figure.

To clarify the nature of the "cross-link" in the PVA gels, X-ray diffraction patterns of water, a PVA gel, and a film were examined. They are shown in Figures $3 a, b$, and c. PVA no. $4(x=1682)$ was used to prepare the gel. The polymer concentration in the gel was $25 \mathrm{~g} / l$, and the film was prepared by drying the gel. The photographs were taken at $15^{\circ} \mathrm{C}$ to prevent gel melting. For water and the gel, only haloes are observed, whereas, for the PVA film, diffraction rings are observed. Obviously, the PVA film is crystalline, and it was found that the $d$-spacings obtained from Figure $3 \mathrm{c}$ are in close agreement with those reported by Mochizuki. ${ }^{18}$ In order to compare the patterns of gel and water, $d$-spacings were calculated by applying Bragg's equation for the peak positions of the haloes. The innermost halo $(d=7.8 \AA)$ of the gel definitely differs in position from the same halo ( $d=5.3 \AA$ ) of water, but other haloes of both the gel and water were almost in the same positions. The $d=7.8 \AA$ halo of the gel is near to the (110) strong diffraction ring $(d=7.08 \AA)$ of the PVA film. However, judging from the comparison of the photographs, and also taking into consideration that the syndiotactic sequence length $=7$ in the "cross-links," we must conclude that the "cross-links" in the PVA gel are not large enough to be observed by X-rays. It is supposed that the "cross-link" consists of syndiotactic sequences of a few PVA molecules adlineated together to form the crystalline nucleus (or embryo crystallite), which then grows upon evaporation of water to form a crystallite large enough to be observed by X-ray diffraction. Of course, hydrogen bonding is important; it is this factor plus geometrical fit, however, which leads to the building of the PVA crystalline structure. ${ }^{19}$ The multiple hydrogen bonds formed from several syndiotactic sequences in a nucleus would be more stable than hydrated bonds.

Acknowledgment. We are indebted to Dr.
Minoru Ueda and Mr. Masaru Kawahashi, Kuraray Co. Ltd, for their kind supply of the PVA samples. Special thanks are due to Professor Ikumi Kagawa for his encouragement throughout this research.

\section{REFERENCES}

1. A. Takahashi, T. Nakamura, and I. Kagawa, Polymer J., 3, 207 (1972).

2. J. E. Eldridge and J. D. Ferry, J. Phys. Chem., 58, 992 (1954).

3. A. Takahashi, Polymer J., 4, 379 (1973).

4. H. Maeda, T. Kawai, and R. Kashiwagi, Kobunshi Kagaku (Chem. High Polymers), 13, 193 (1956).

5. N. Hirai in "Poly(vinyl alcohol)" (Collection of Papers for the First Symposium on PVA, Osaka, 1955), I. Sakurada Ed., Society of Polymer Science, Japan, p 325.

6. H. C. Haas and R. McDonald, J. Polym. Sci., Part A-1, 10, 1617 (1972).

7. H. Maeda, T. Kawai, and R. Kashiwagi, Kogyo Kagaku Zashi (J. Chem. Soc. Japan, Ind. Chem. Sect.), 59, 809 (1956).

8. K. Shibatani, Polymer J., 1, 348 (1970).

9. A. Nakajima and K. Furudachi, Kobunshi Kagaku (Chem. High Polymers), 6, 460 (1949).

10. S. Murahashi, S. Nozakura, M. Sumi, H. Yuki, and K. Hatada, ibid., 23, 605 (1966).

11. T. Ito, K. Noma, and I. Sakurada, ibid., 16, 115 (1959).

12. P. J. Flory, "Principles of Polymer Chemistry," Cornell University Press, Ithaca, N.Y., 1953, Chapter XII.

13. H. A. Dieu, J. Polym. Sci., 12, 417 (1954).

14. I. Sakurada, A. Nakajima, and H. Takita, Kobunshi Kagaku (Chem. High Polymers), 11, 21 (1954).

15. L. Pauling, "The Nature of the Chemical Bond" 3rd ed, Cornell University Press, Ithaca, N.Y., 1960, Chapter XII.

16. M. Sumi, K. Matsumura, R. Ohno, S. Nozakura, and S. Murahashi, Kobunshi Kagaku (Chem. High Polymers), 24, 606 (1957).

17. S. Murahashi, H. Yuki, T. Sano, U. Yonemura, H. Tadokoro, and Y. Chatani, J. Polym. Sci., 62, 577 (1962).

18. T. Mochizuki, Nippon Kagaku Zashi, 81, 15 (1960).

19. R. Hill, "Fibres from Synthetic Polymers," Elsevier Pub. Co., Amsterdam, 1953, Chapter XIII. 